\title{
Validation of fetus aneuploidy in 221 Korean clinical samples using noninvasive chromosome examination: Clinical laboratory improvement amendments- certified noninvasive prenatal test
}

Min-Jeong Kim, Chang Hyuk Kwon, Dong-In Kim, Hee Su Im, Sungil Park, Ji Ho Kim, Jin-Sik Bae, Myunghee Lee, and Min Seob Lee* Eone-Diagnomics Genome Center (EDGC), Inc., Incheon, Korea

Purpose: We developed and validated a fetal trisomy detection method for use as a noninvasive prenatal test (NIPT) including a Clinical Laboratory Improvement Amendments (CLIA)-certified bioinformatics pipeline on a cloud-based computing system using both Illumina and Life Technology sequencing platforms for 221 Korean clinical samples. We determined the necessary proportions of the fetal fraction in the cell-free DNA (cfDNA) sample for NIPT of trisomies 13, 18, and 21 through a limit of quantification (LOQ) test.

Materials and Methods: Next-generation sequencing libraries from 221 clinical samples and three positive controls were generated using Illumina and Life Technology chemistries. Sequencing results were uploaded to a cloud and mapped on the human reference genome (GRCh37/hg19) using bioinformatics tools. Based on Z-scores calculated by normalization of the mapped read counts, final aneuploidy reports were automatically generated for fetal aneuploidy determination.

Results: We identified in total 29 aneuploid samples, and additional analytical methods performed to confirm the results showed that one of these was a false-positive. The LOQ test showed that the proportion of fetal fraction in the cfDNA sample would affect the interpretation of the aneuploidy results.

Conclusion: Noninvasive chromosome examination (NICE), a CLIA-certified NIPT with a cloud-based bioinformatics platform, showed unambiguous success in fetus aneuploidy detection.

Key words: Prenatal diagnosis, High-throughput nucleotide sequencing, Computational biology, Cell-free DNA, Trisomy, Aneuploidy.

\section{Introduction}

Recent technological advancements in next generation sequencing (NGS) and bioinformatics have led to a novel noninvasive prenatal test (NIPT) for analyzing fetus aneuploidy using cell-free DNA (cfDNA) in the plasma of pregnant women. Whereas other NIPT technologies perform an analysis of specific regions of the chromosome by a targeted sequencing method, such as single nucleotide polymorphism genotyping or haplotype-based analysis [1-3], this novel chromosome

\footnotetext{
Received: 11 November 2015, Revised: 14 December 2015, Accepted: 24 December 2015, Published: 31 December 2015

${ }^{*}$ Corresponding author: Min Seob Lee, Ph.D.

Eone-Diagnomics Genome Center (EDGC), Inc., 291 Harmony-ro, Yeonsu-gu, Incheon 22014, Korea.

Tel: +82-32-210-2341, Fax: +82-32-210-2349, E-mail: mlee@edgc.com

Conflict of interest: The authors of this publication is working in the Eone-Diagnomics Genome Center which is developing products related to the research being reported.

(C) This is an open-access article distributed under the terms of the Creative Commons Attribution Non-Commercial License (http://creativecommons.org/licenses/by-nc/4.0/) which permits unrestricted non-commercial use, distribution, and reproduction in any medium, provided the original work is properly cited.

(c) Copyright 2015 by the Korean Society of Medical Genetics 
analysis uses massive parallel sequencing data obtained by an NGS technology and applies statistical normalization to each chromosome read count $[4,5]$. Sequencing reads are mapped to the human reference genome (GRCh37/hg19) and quantified according to their genomic locus. After normalizing the read count in a specific bin of a genomic region, Z-scores are calculated for each chromosome to determine fetal aneuploidy status.

We developed the noninvasive chromosome examination (NICE) test to improve the processing and success rate of the NIPT, and we implemented the data processing, report generation, and file storage on a secure, cloud-based platform. The NICE process, including the cloud-based bioinformatics and reporting system, was developed to meet the quality control requirements of clinical laboratory standards and has been fully validated and accredited under the Clinical Laboratory Improvement Amendments (CLIA) in the United States.

In the present study, we performed a validation of the new test by comparing the outcome of a NICE analysis to the known results of 221 clinical samples and three proprietary control samples we developed. We generated 227 NGS sequencing libraries using Illumina and Life Technology sequencing chemistries, including two libraries for each three positive aneuploid controls, and we then tested and validated NICE using the automated cloud-based bioinformatics platform.

\section{Materials and Methods}

\section{Cell-free DNA extraction from maternal blood}

Blood samples $(10 \mathrm{~mL})$ were collected from 221 participants into Cell-Free DNA BCT tubes (Streck, Omaha, NE, USA) and centrifuged at $1,800 \times g$ for $15 \mathrm{~min}$ at $4^{\circ} \mathrm{C}$. Plasma samples were transferred into $1.7-\mathrm{mL}$ tubes and then centrifuged at $18,000 \times g$ for $15 \mathrm{~min}$ at $4^{\circ} \mathrm{C}$. The separated plasma samples were transferred to new tubes and stored at $-80^{\circ} \mathrm{C}$. cfDNA was isolated from $2 \mathrm{~mL}$ plasma by using the OIAamp Circulating Nucleic Acid Kit (Qiagen, Hilden, Germany) according to the manufacturer's instructions.

\section{Next-generation sequencing and data analysis}

Isolated cfDNA samples ( $<100 \mathrm{ng}$ ) were used as input DNA for either Illumina or Life Technology sequencing reactions. HiSeq 100-bp paired-end (PE), 50-bp single-read (SR), or Proton SR sequencing libraries were prepared according to the manufacturer's instructions (IIlumina, San Diego, CA, USA; Life Technology, Carlsbad, CA, USA). All sequencing data were then uploaded to the cloud-based bioinformatics platform to perform the aneuploidy test and to generate results based on Z-score calculations for review.

First, we mapped the sequencing reads to the human reference genome (GRCh37/hg 19) using Burrows-Wheeler Aligner (BWA) software; we then sorted the mapped reads and normalized the read count using Samtools. This was followed by detection and removal of duplicated reads. Finally, we calculated the Z-score with a GC correction and mapping correction using the ReadDepth R module [6-8]. Upon approval of the CLIA director, final reports were automatically generated from the NICE portal (Fig. 1).

\section{Results}

\section{Limit of quantification (LOO) test}

Although NGS-based NIPT aneuploidy tests have high sensitivities and specificities, the accuracy of each test is heavily influenced by the amount of fetal DNA present in maternal plasma. A LOQ test is very important to assess the reliable lowest quantity of fetal DNA in a sample to be differentially detected because the concentration of fetal DNA in maternal plasma can vary depending on maternal health factors, gestational age, and health of the placenta $[9,10]$. In order to evaluate the sensitivity of the NIPT, an LOQ test is essential for the analytical procedure.

For the test, we used non-fetal mother (Cat. No. AG09387), T13 (GM02948), T18 (GM01359), or T21 (AG09394) cell lines purchased from the Coriell Institute for Medical Research (Camden, NJ, USA). The LOQ test was performed with different

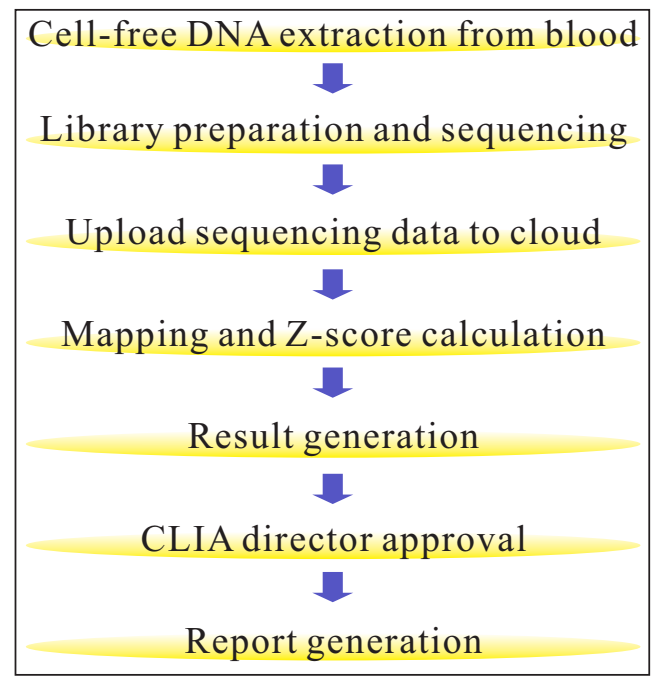

Fig. 1. Noninvasive chromosome examination (NICE) work flow. CLIA, Clinical Laboratory Improvement Amendments. 
concentrations of trisomy genomic DNA samples extracted from the cells, and each DNA sample was mixed with the genomic DNA solution of the non-fetal mother in concentrations of : $0.5 \%, 2 \%, 4 \%, 6 \%, 8 \%$, and $10 \%$ fetal DNA. We also prepared a DNA sample extracted from the normal brother (AG09392) of T21 as a negative control and used the same serial concentrations. Three replicated NGS libraries for each sample were prepared, and sequencing data for all 72 libraries were generated using a Proton sequencer (Life Technology).

From the results of mapping the sequencing data to the human genome (see Materials and Methods), LOQ was calculated with a 95\% confidence level based on the International Council for Harmonisation of Technical Requirements for Pharmaceuticals for Human Use (ICH) guidelines. We found that the proportion of trisomy 13 fetal DNA should be more than $2.5 \%$ of the sample to detect T13 aneuploidy. More than $4.1 \%$ and $5.2 \%$ of the chromosome 18 and 21 trisomies in the samples could be detected, respectively, meaning that lower concentrations of trisomy DNA in the samples may lead to false negatives on the aneuploidy test (Fig. 2).

\section{Cloud-based clinical aneuploidy test}

In total, 221 maternal blood samples were obtained from voluntary participants, and three different cell lines of chromosome 13, 18, and 21 trisomies were used as positive controls. Among the pregnant participants, 207 cases were categorized as high-risk based on previous tests and/or classifications including 137 cases of positive serum screening, 41 cases of advanced maternal age, five cases of abnormal ultrasonography, four cases of genetic history, and 20 cases of multiple indications of the above. Mean maternal age was 33.2 years, ranging from 26 to 40 years, and most samples (80.5\%) were collected during the second trimester (Table 1). This study was approved in September 2013 by the Eone Laboratories Institutional Review Board (IRB \# 128477-201309-BR-001-01). Informed consent was obtained from the participants for the study based on IRB guidelines.

The 221 cfDNA and six genomic DNA reference samples were extracted from the participants' blood and cultured three positive control cells, respectively. The Illumina HiSeq NGS platform was used to generate 100-bp PE or 50-bp SR sequences from 50 and 20 cfDNA samples, respectively, while 151 cfDNA and six control DNA samples were processed with the Proton Life Technology NGS platform to generate SR sequencing reads. Each sequencing dataset was transferred to the cloud to perform the NICE aneuploidy test (Fig. 3).
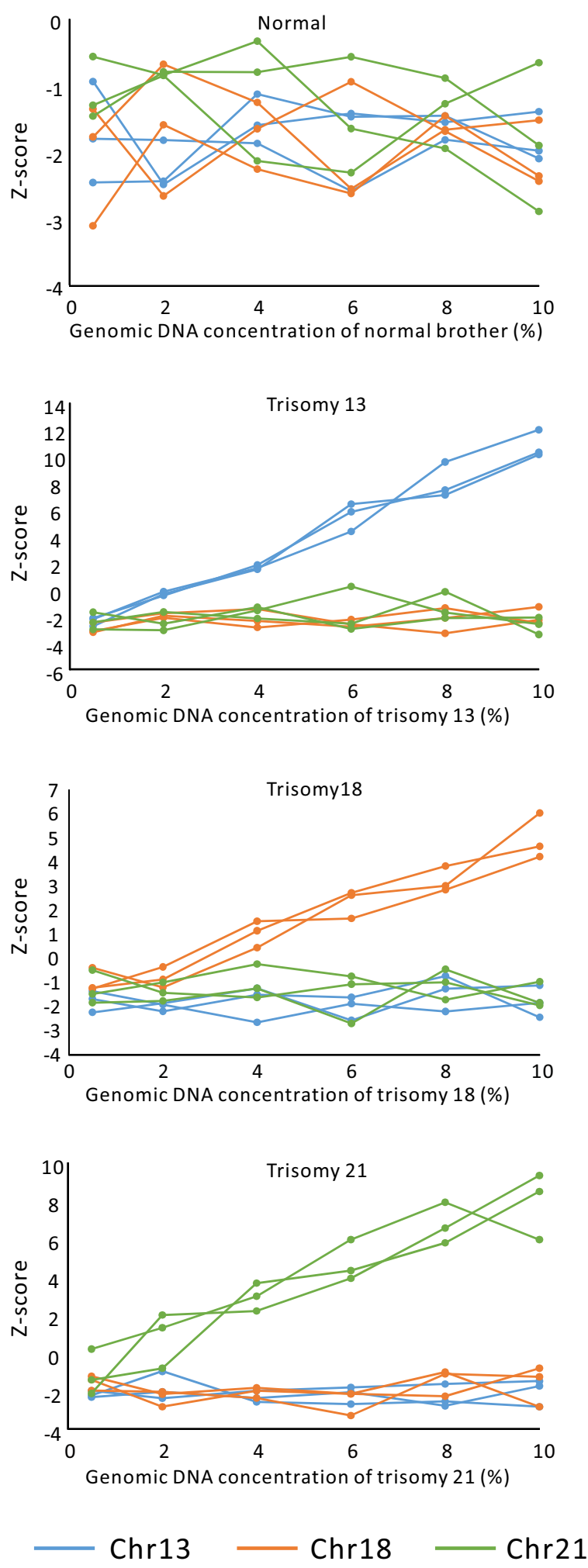

Fig. 2. Limit of quantitation test with negative and positive aneuploidy samples. Trisomy genomic DNA samples were mixed with the genomic DNA solution of the non-fetal mother in concentrations of: $0.5 \%, 2 \%$, $4 \%, 6 \%, 8 \%$, and $10 \%$. Z-scores of each 13,18 and 21 chromosome are shown. (A) Normal brother of T21. (B) Trisomy 13. (C) Trisomy 18. (D) Trisomy 21. 
Table 1. Demographic information for the pregnant participants of the NICE test

\begin{tabular}{|c|c|c|c|c|}
\hline \multirow{2}{*}{ Variable } & \multirow{2}{*}{ Sample size (n) } & \multicolumn{3}{|c|}{ Number of samples with aneuploidy } \\
\hline & & Trisomy 21 & Trisomy 18 & Trisomy 13 \\
\hline \multicolumn{5}{|l|}{ Maternal age (yr) } \\
\hline 26 & 8 & 0 & 0 & 0 \\
\hline 27 & 14 & 2 & 1 & 0 \\
\hline 28 & 16 & 2 & 1 & 0 \\
\hline 29 & 16 & 0 & 0 & 0 \\
\hline 30 & 17 & 1 & 0 & 0 \\
\hline 31 & 15 & 2 & 1 & 1 \\
\hline 32 & 11 & 1 & 0 & 0 \\
\hline 33 & 16 & 2 & 0 & 0 \\
\hline 34 & 17 & 1 & 0 & 0 \\
\hline 35 & 15 & 0 & 0 & 0 \\
\hline 36 & 16 & 0 & 1 & 0 \\
\hline 37 & 16 & 2 & 2 & 0 \\
\hline 38 & 16 & 1 & 0 & 0 \\
\hline 39 & 16 & 2 & 0 & 0 \\
\hline 40 & 12 & 6 & 0 & 0 \\
\hline \multicolumn{5}{|l|}{ Gestational age at test } \\
\hline First trimester (10-13 weeks) & 29 & 3 & 1 & 0 \\
\hline Second trimester (14-27 weeks) & 186 & 19 & 5 & 1 \\
\hline Third trimester ( $\geq 28$ weeks) & 5 & 0 & 0 & 0 \\
\hline Unknown & 1 & 0 & 0 & 0 \\
\hline \multicolumn{5}{|l|}{ Test platform } \\
\hline Illumina (PE or SR) ${ }^{\mathrm{a}}$ & 70 & 10 & 5 & 0 \\
\hline Proton (SR) & 151 & 12 & 1 & 1 \\
\hline Additional test for NICE-positive samples ${ }^{\mathrm{a}}$ & 29 & 22 & 6 & 1 \\
\hline
\end{tabular}

NICE, noninvasive chromosome examination; PE, paired-end; SR, single-read.

${ }^{a}$ Amniocentesis, quantitative fluorescent polymerase chain reaction (QF PCR), fluorescence in situ hybridization (FISH) and/or Illumina Verifi.

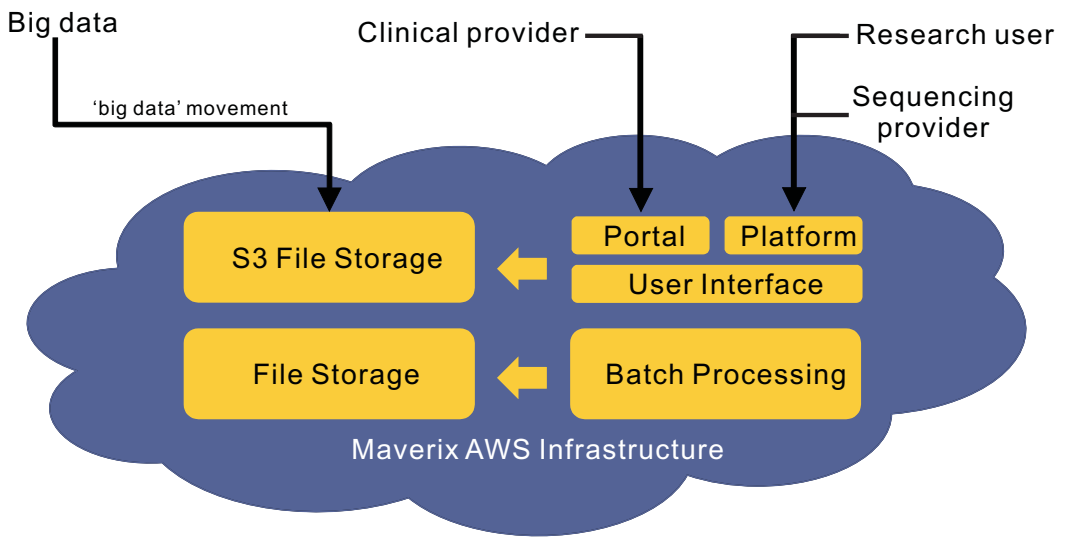

Fig. 3. High-level cloud architecture of noninvasive chromosome examination (NICE) test. AWS, Amazon web service.
Of the 221 samples, 29 exhibited aneuploidy based on our Z-score criteria: 22 for trisomy 21, 6 for trisomy 18, and 1 for trisomy 13 (Table 1 and Fig. 3). After the genetic consultation, additional tests for 21 samples including amniocentesis, quantitative fluorescent polymerase chain reaction (QF PCR), and/or fluorescence in situ hybridization (FISH), were performed to validate the aneuploidy results. All were consistent with the NICE results except for one trisomy 21 sample (\#25) (Table 2), 
Table 2. Additional tests performed for the samples showing NICE aneuploidy results

\begin{tabular}{|c|c|c|c|c|c|}
\hline Sample & NICE & Amniocentesis & QF PCR & FISH & Verifi \\
\hline 1 & $\mathrm{~T} 13$ & T13 & $-a^{a}$ & - & $\mathrm{T} 13$ \\
\hline 2 & T18 & T18 & T18 & T18 & - \\
\hline 3 & T18 & T18 & - & - & Hemolysis \\
\hline 4 & T18 & T18 & T18 & - & - \\
\hline 5 & T18 & T18 & - & - & - \\
\hline 6 & T18 & T18 & T18 & - & - \\
\hline 7 & T18 & NT & - & - & T18 \\
\hline 8 & T21 & T21 & T21 & - & - \\
\hline 9 & T21 & $\mathrm{T} 21$ & - & T21 & - \\
\hline 10 & T21 & T21 & - & - & - \\
\hline 11 & $\mathrm{~T} 21$ & $\mathrm{~T} 21$ & $\mathrm{~T} 21$ & - & - \\
\hline 12 & T21 & T21 & T21 & T21 & - \\
\hline 13 & T21 & $\mathrm{T} 21$ & - & - & - \\
\hline 14 & T21 & T21 & - & - & - \\
\hline 15 & T21 & T21 & - & - & - \\
\hline 16 & T21 & T21 & $\mathrm{T} 21$ & - & - \\
\hline 17 & T21 & $\mathrm{T} 21$ & T21 & - & - \\
\hline 18 & T21 & T21 & T21 & - & - \\
\hline 19 & $\mathrm{~T} 21$ & $\mathrm{~T} 21$ & T21 & - & - \\
\hline 20 & $\mathrm{~T} 21$ & - & - & - & $\mathrm{T} 21$ \\
\hline 21 & T21 & - & - & - & $\mathrm{T} 21$ \\
\hline 22 & T21 & - & - & - & T21 \\
\hline 23 & T21 & - & - & - & T21 \\
\hline 24 & T21 & - & - & - & T21 \\
\hline 25 & T21 & Negative & Negative & Negative & T21 \\
\hline 26 & T21 & T21 & - & - & T21 \\
\hline 27 & T21 & $\mathrm{T} 21$ & - & - & T21 \\
\hline 28 & T21 & - & - & - & T21 \\
\hline 29 & $\mathrm{~T} 21$ & - & - & - & $\mathrm{T} 21$ \\
\hline
\end{tabular}

NICE, noninvasive chromosome examination; QF PCR, quantitative fluorescent polymerase chain reaction; FISH, fluorescence in situ hybridization.

Blank cells indicate 'not-tested'.
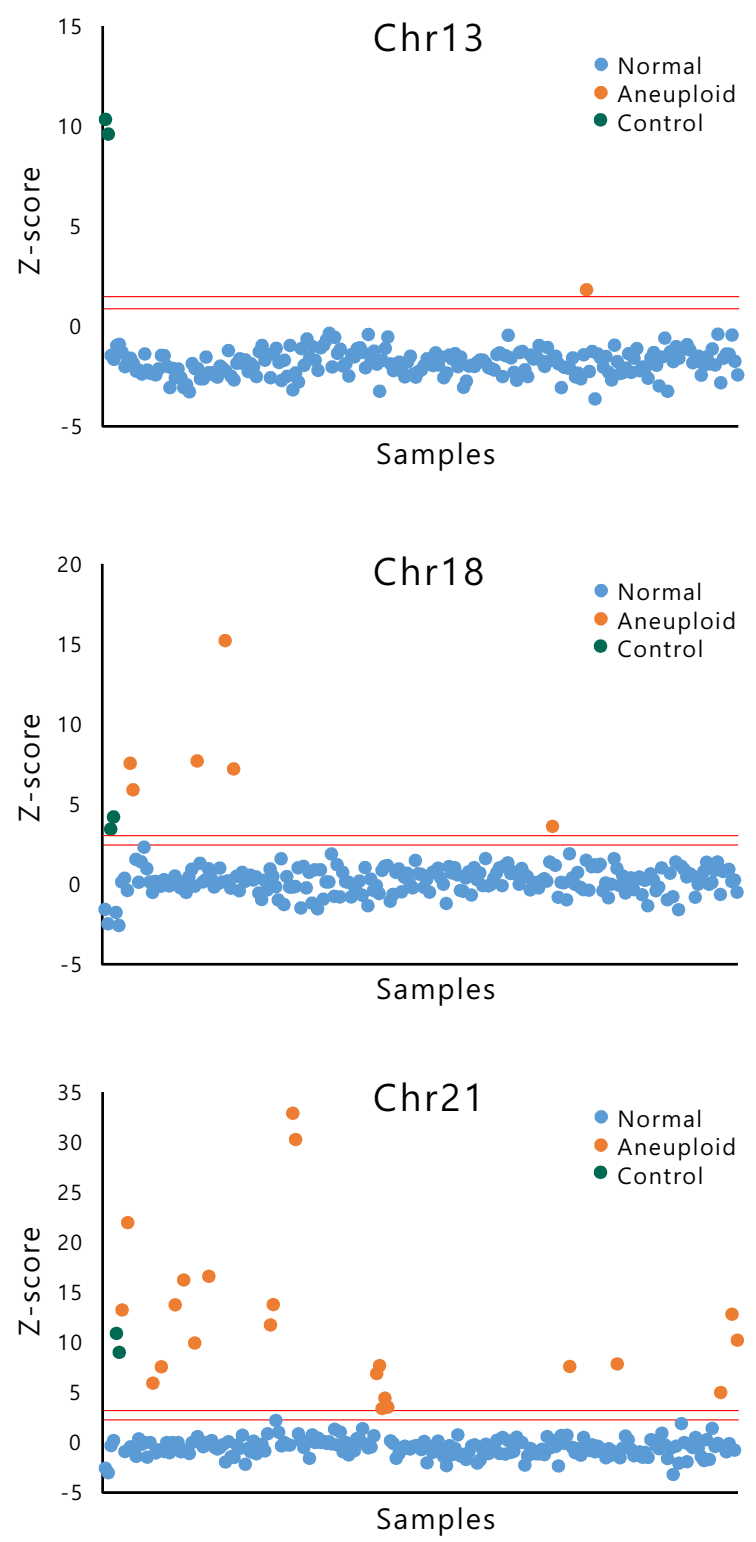

Fig. 4. Z-scores of the 221 clinical and six positive control samples.

Table 3. Validation test comparing NICE results with amniocentesis

\begin{tabular}{ccccrccc}
\hline Trisomy & N & Sensitivity (\%) & $95 \% \mathrm{Cl}$ & Specificity (\%) & $95 \% \mathrm{Cl}$ & Incidence $(\%)$ \\
\hline T13 & 81 & $100(1 / 1)$ & $2.50-100$ & $100(80 / 80)$ & $95.49-100$ & 1.23 \\
T18 & 81 & $100(5 / 5)$ & $47.82-100$ & $100(76 / 76)$ & $95.26-100$ & 6.17 \\
T21 & 81 & $100(14 / 14)$ & $76.84-100$ & $98.51(66 / 67)$ & $91.96-99.96$ & 17.28 \\
Total & 81 & $100(20 / 20)$ & $83.16-100$ & $98.36(60 / 61)$ & $91.20-99.96$ & 24.69 \\
\hline
\end{tabular}

NICE, noninvasive chromosome examination; $\mathrm{Cl}$, confidence interval.

which was confirmed as normal by amniocentesis, QF PCR, and FISH. However, interestingly, an Illumina Verifi test for the sample reported the same result as the NICE test. All positive controls were clearly detected in the aneuploidy Z-score region
(Fig. 4). Additionally, among the 192 women classified as NIPTnegative, 64 underwent further aneuploidy testing, including amniocentesis, QF PCR, and/or FISH; all were confirmed to be negative for aneuploidy. As a result, we compared 81 NICE 
validation tests with amniocentesis results, as amniocentesis has been accepted as the gold standard for diagnosing aneuploidy (Table 3). There were 20 true positives and one false positive case in this test, resulting in a test sensitivity of 100\% and specificity of $98.36 \%$, and the incidences of trisomies 13, 18, and 21 in the sample were $1.23 \%, 6.17 \%$, and $17.28 \%$, respectively.

We also performed a proficiency test to assess how well our data is analyzed compared to the Verifi NIPT results for 129 samples. Among 14 aneuploid samples based on Verifi results, 13 were detected as aneuploid based on the NICE test, all of which were consistent with amniocentesis results. All of the results for the negative-aneuploidy samples using NICE were also consistent with Verifi.

\section{Discussion}

Due to its noninvasiveness, superior sensitivity and specificity, and higher success rate compared to conventional aneuploidy screening methods, NGS-based NIPT has been widely accepted for prenatal aneuploidy tests. We developed the NICE procedure and implemented the pipeline on a cloud-based system. We successfully identified trisomy 13 (Patau), 18 (Edward), and 21 (Down) syndromes in 221 clinical samples and three trisomy positive controls using the cloud-based pipeline.

In total, we found 22 cases of trisomy 21, six cases of trisomy 18 , and one case of trisomy 13 . These results were verified with other conventional aneuploidy tests. Only one of the positive samples was found to be a false-positive upon comparison with the amniocentesis result. Of the 81 clinical samples that were compared to the amniocentesis results, we were able to successfully classify 80 NIPT samples (98.77\%), including 59 normal and 21 aneuploidy cases, due to the high performance of our CLIA-certified NICE pipeline. Positive controls were also clearly detected using the same pipeline. An LOO test showed the importance of the proportion of the fetal fraction in the sample, indicating that an insufficient amount of fetal DNA would lead to a false interpretation of aneuploidy. Further efforts are needed to develop a method of examining the amount of fetal DNA in maternal plasma in order to avoid misinterpretation.

The NICE analysis pipeline and reporting system were implemented on a secure Amazon cloud-based system, and the whole pipeline was certified under the CLIA in the United States. The clinically approved NICE cloud system offers an opportunity to perform clinical sample testing under the CLIA guidance to the labs which want to provide the NIPT service.

\section{Acknowledgements}

The authors would like to express appreciation to the patients who donated blood and to Eone reference laboratory for collecting samples for the study. We also thank the team at Maverix Biomics for helping with cloud preparation and ResearchDx for CLIA consultation.

\section{References}

1. Zimmermann B, Hill M, Gemelos G, Demko Z, Banjevic M, Baner J, et al. Noninvasive prenatal aneuploidy testing of chromosomes 13,18 , $21, X$, and $Y$, using targeted sequencing of polymorphic loci. Prenat Diagn 2012;32:1233-41.

2. Curnow KJ, Wilkins-Haug L, Ryan A, Kırkızlar E, Stosic M, Hall MP, et al. Detection of triploid, molar, and vanishing twin pregnancies by a single-nucleotide polymorphism-based noninvasive prenatal test. Am J Obstet Gynecol 2015;212:79.e1-9.

3. Xu Y, Li X, Ge HJ, Xiao B, Zhang YY, Ying XM, et al. Haplotype-based approach for noninvasive prenatal tests of Duchenne muscular dystrophy using cell-free fetal DNA in maternal plasma. Genet Med 2015;17:889-96.

4. Fan HC, Blumenfeld YJ, Chitkara U, Hudgins L, Quake SR. Noninvasive diagnosis of fetal aneuploidy by shotgun sequencing DNA from maternal blood. Proc Natl Acad Sci U S A 2008;105:16266-71.

5. Chiu RW, Chan KC, Gao Y, Lau VY, Zheng W, Leung TY, et al. Noninvasive prenatal diagnosis of fetal chromosomal aneuploidy by massively parallel genomic sequencing of DNA in maternal plasma. Proc Natl Acad Sci U S A 2008;105:20458-63.

6. Li H, Durbin R. Fast and accurate short read alignment with BurrowsWheeler transform. Bioinformatics 2009;25:1754-60.

7. Li H, Handsaker B, Wysoker A, Fennell T, Ruan J, Homer N, et al; 1000 Genome Project Data Processing Subgroup. The Sequence Alignment/ Map format and SAMtools. Bioinformatics 2009;25:2078-9.

8. Miller CA, Hampton O, Coarfa C, Milosavljevic A. ReadDepth: a parallel $\mathrm{R}$ package for detecting copy number alterations from short sequencing reads. PLoS One 2011;6:e16327.

9. Vora NL, Johnson KL, Basu S, Catalano PM, Hauguel-De Mouzon $\mathrm{S}$, Bianchi DW. A multifactorial relationship exists between total circulating cell-free DNA levels and maternal BMI. Prenat Diagn 2012;32:912-4.

10. Levine RJ, Qian C, Leshane ES, Yu KF, England $\amalg$, Schisterman EF, et al. Two-stage elevation of cell-free fetal DNA in maternal sera before onset of preeclampsia. Am J Obstet Gynecol 2004;190:707-13. 